\title{
Epidemiological, Clinical, Laboratorial and CT Imaging Features of Hospitalized Patients with Initially Negative Detection of SARS-CoV-2 in China
}

\author{
Yingjian Liang \\ Xiaobin Zheng (D) \\ Meizhu Chen (D) \\ Changli Tu \\ Cuiyan Tan \\ Yiying Huang \\ Zhenguo Wang (D) \\ Jian $\mathrm{Wu}$ \\ Jing Liu
}

Department of Pulmonary and Critical Care Medicine (PCCM), The Fifth Affiliated Hospital of Sun Yat-sen University, Zhuhai, People's Republic of China
Correspondence: Jing Liu; Xiaobin Zheng Department of Pulmonary and Critical Care Medicine (PCCM), The Fifth Affiliated Hospital of Sun Yat-sen University, 52 East Meihua Road, Zhuhai City, 519000, People's Republic of China Tel/Fax +867562528733

Email liujing25@mail.sysu.edu.cn;

zhxbin@mail.sysu.edu.cn
Purpose: The main objective of this study was to decipher the general epidemiology, clinical characteristic, laboratory finding and chest computed tomography (CT) imaging features of the novel coronavirus disease (COVID-19) patients whose initial detection of severe acute respiratory syndrome coronavirus-2 (SARS-CoV-2)is negative.

Methods: Nearly 100 confirmed cases admitted to The Fifth Affiliated Hospital of Sun Yatsen University from 18th January to 26th February, 2020, were screened. Clinical records, laboratory results and CT images were reviewed for nine COVID-19 patients with initially negative RT-PCR detection.

Results: Fever and cough were common, and one patient merely present gastrointestinal symptoms. Increasing CRP and decreasing ALB were showed in nearly half of the patients among negative detection and return to normal level after real time polymerase chain reaction (RT-PCR) results converted from positive to negative. Left lower lobe was affected nearly in all the patients. A patient received oxygen support timely according his high Mulbsta score.

Conclusion: Our study elucidated on the clinical features of hospitalized patients with initially negative detection of SARS-CoV nucleic acid. Patient merely with symptoms associated with digestive system should be screened for COVID-19. CT scan and repeated RT-PCR are two powerful diagnostic tools. Mulbsta score assessing in the early stage enhances the confidence of severity evaluation in physician.

Keywords: COVID-19, negative detection, clinical characteristic, laboratory finding, CT images features

\section{Introduction}

The novel coronavirus disease (COVID-19) has spread rapidly and raised worldwide concern as an increasing global health burden. The pathogen of COVID-19 was identified as a novel beta-coronavirus named severe acute respiratory syndrome coronavirus-2 (SARS-CoV-2) later. Whole genome sequencing analysis as well as phylogenetic analysis revealed that SARS-CoV-2 differed from two notorious family members, Middle East respiratory syndrome coronavirus (MERS-CoV) and severe acute respiratory syndrome coronavirus (SARS-CoV). ${ }^{1}$ The genome SARS-CoV showed high identity with Bat-Cov, which indicated that bats may be the reservoir host for SARS-CoV and a recent report suggested that Malayan 
pangolins had the potential to act intermediate host of SARS-CoV as it highly related amino acid identity in the E, M, N and S genes. ${ }^{2,3}$ Screening of the pathogen by nucleic acid testing like real time polymerase chain reaction (RT-PCR) is an essential diagnostic standard of COVID-19. Angiotensin-converting enzyme 2 (ACE-2), a receptor widely present in respiratory as well as gastrointestinal tract, mainly mediates the entry of SARS-CoV-2 into human cells, and consequently upper or lower respiratory samples like nasal swab or throat swab as well as gastrointestinal like fecal swab or stool are the major type of testing. ${ }^{4}$ However, in the early stage of COVID-19, load of SARS-CoV-2 from respiratory or gastrointestinal system may be lower than detection limit. It has been reported that nearly $10 \%(10 / 102)$ RT-PCR was negative initially. ${ }^{5}$ Therefore, a proportion of the COVID-19 patients whose primary screening of SARS-CoV-2 nucleic acid showed negative but confirmed later. It's important to recognize the patient as early as possible to avoid transmitting virus. It is necessary to know about the specific information including the general epidemiology, clinical characteristics, laboratory finding and computed tomography (CT) imaging features about them. Herein, we present nine patients whose SARS-CoV-2 nucleic acid detection was negative initially but converted later and our aim is to achieve a better understanding of the COVID-19 virus.

\section{Method}

\section{Study Design and Participants}

Our study was conducted in accordance with the Declaration of Helsinki and was approved by the Research Ethics Committee of the Fifth Affiliated Hospital of Sun Yat-sen University (Ethics approval number K30-1). Written informed consent for this retrospective study was waived by our institutional review board because of no potential risk to patients. Anonymous data was collected and analyzed by researchers who had no available link to the patients.

\section{Data Collection}

We screened 98 confirmed cases admitted to The Fifth Affiliated Hospital of Sun Yat-sen University, one of the biggest tertiary hospital serving for millions people in Guangdong Province from 18th January to 26th February, 2020. All of them were identified with positive results by RT-PCR from Chinese Center for Disease Control and Prevention (CDC) recommended Kit
(BioGerm, Shanghai, China), following WHO guidelines. Samples were from the upper respiratory tract, such as a throat or nasal swab, as well as gastrointestinal, such as a fecal swab or stool sample. Only the patients who provided a negative nucleic acid test initially, but tested positive later, were taken into consideration.

We carried out a retrospective review of their clinical records, laboratory testing and chest CT scans at three time points according to the result of RT-PCR of throat or nasal swab, which including negative detection (stage 1), firstly positive detection (stage 2) and negative converting (stage 3). The clinical records were reviewed by two respiratory professors, including medical history, smoking history, exposure history, duration of symptoms, oxygen therapy. Two investigators independently recorded the laboratory finding, including white blood cell count, neutrophil count, lymphocyte count, eosinophil count, C-reactive protein (CRP), alanine aminotransferase (ALT), aspartate aminotransferase (AST), lactate, serum albumin (ALB), seroglobulin (GLB), serum creatinine, creatine kinase and partial arterial oxygen pressure $\left(\mathrm{PaO}_{2}\right)$. In addition, the number of days between symptomology onset and the date of first negative as well as positive detection was tracked. CT images were described by two experienced specialists independently, and were evaluated including ground glass opacity (GGO), crazypaving pattern, consolidation, pleural effusion and absorbed according to Fleischner Society glossary and peer-reviewed literature about viral pneumonia. ${ }^{6}$ The distribution of lung lobes involved was described as sub pleural, central, random or diffuse. The Mulbsta Score was also used as routine evaluation. ${ }^{7}$

\section{Statistical Analysis}

Statistical analysis was performed using SPSS (version 20; IBM, New York, USA). Quantitative variables were presented as mean \pm standard deviation and the categorical variables were expressed as the percentage of the whole (\%).

\section{Result}

\section{Baseline Data}

Of nearly 100 confirmed cases screened, only 4 males and 5 females met the standard and were assessed in this study. The age range was from 1 to 64 years $(33.22 \pm 18.14)$. No smokers were found. One of them had the history of hypertension and three of them had the history of chronically gastrointestinal diseases. A young woman has 
a history of eczema. Nobody had an underlying history of cancer or diabetes. Seven of them had travelled to Wuhan city and five of them had exposure to the COVID-19 patients. One young man had not stayed in Wuhan or had contact with the infectious. The average days between the symptom onset and an initially positive RT-PCR detection 6.22 (range from 2 to 10 ) while the negative result is 2.44 (range from 1 to 7). Range of the positive results' duration from upper respiratory sample was 1 to 20 (5.56 \pm 6.06 ). Mean value of the fecal swab or stool was longer (6.56) (Table 1). Three patients' stool still indicated a positive state (Figure 1).

\section{Clinical Symptoms}

Regarding the clinical symptoms during stage 1, six of nine patients present fever and three of them had co-existed cough. Although five patients present cough, there was no expectoration. Chest tightness was also observed in two of them. Additionally, the one had unknown exposure history showed obvious nausea and diarrhea without fever or respiratory symptoms. In stage 2 , no fever but cough was still present. One of them developed to dyspnea. No symptoms by one cases still showed cough in stage 3. Only patient 4's Mulbsta Score was higher than 12 (Table 2).

\section{Laboratory Finding}

Patient 6 and patient 7 were admitted in other hospital at first with a negative result of the SARS-CoV-2 but they were transmitted to our hospital as high suspicions of COVID-19 for the exposure history or CT scan. Consequently, laboratory find before our hospital admitting of stage 1 were not collected. Other data from laboratory finding exhibited that two patients had elevated concentrations of CRP $(>10 \mathrm{mg} / \mathrm{L})$ in stage 1. No leukopenia or leukocytosis was showed among the patients. Range of lymphocyte count was $0.71-4.96$ $\mathrm{X} 10^{\wedge} 9 / \mathrm{L}$. Average level of ALB was lower than the normal $(40 \mathrm{~g} / \mathrm{L})$. In stage 2 , we found that nearly half of the patient (4/ 9) had increased CRP and decreased ALB. Only two of them showed lymphopenia $\left(<1 \mathrm{X} 10^{\wedge} 9 / \mathrm{L}\right)$. Patient 1 and patient 6 were not available for blood test in stage 3 because of medical requirement. In stage 3 , all the CRP and lymphocyte level return to normal. There is a 5 times higher in the eosinophil concentrations compared with stage 1 (form 0.08 $\mathrm{X} 10^{\wedge} 9 / \mathrm{L}$ to $0.4 \mathrm{X} 10^{\wedge} 9 / \mathrm{L}$ ). Patient 8 showed high ALT in stage 3. Low level of ALB still persisted and the average level dropped from 40.39 to $39.66 \mathrm{~g} / \mathrm{L}$. The ratio between neutrophil and lymphocyte is similar in stage 1 and stage 2 but increased in stage 3 . The mean concentration of serum
Table I Basic Characteristic

\begin{tabular}{|c|c|}
\hline & ALL \\
\hline \multicolumn{2}{|l|}{ Gender } \\
\hline Male & 5 \\
\hline Female & 4 \\
\hline Age & $33.22 \pm 18.14$ \\
\hline $1 \sim 18$ & I \\
\hline $18-30$ & 4 \\
\hline $31-60$ & 3 \\
\hline $60+$ & I \\
\hline Smoking History & None \\
\hline \multicolumn{2}{|l|}{ Exposure History } \\
\hline Wuhan Exposure & 7 \\
\hline COVID-19 Patient Exposure & 5 \\
\hline Unknown & 1 \\
\hline $\begin{array}{l}\text { Means Days Between Symptoms Onset And } \\
\text { Initial Negative Detection }\end{array}$ & $2.44 \pm 2.01$ \\
\hline $\begin{array}{l}\text { Means Days Between Symptoms Onset And } \\
\text { Initial Positive Detection }\end{array}$ & $6.22 \pm 4.6$ \\
\hline $\begin{array}{l}\text { Duration Of Positive Detection From Upper } \\
\text { Respiratory Samples }\end{array}$ & $5.56 \pm 6.06$ \\
\hline $\begin{array}{l}\text { Duration Of Positive Detection From } \\
\text { Gastrointestinal Samples }\end{array}$ & $6.56 \pm 7.11$ \\
\hline Mean Number Of Detection Before Positive & $1.44 \pm 1.01$ \\
\hline \multicolumn{2}{|l|}{ Previous Medical History } \\
\hline Diabetes & 0 \\
\hline Hypertension & I \\
\hline Cancer & 0 \\
\hline Gastrointestinal Disease & 3 \\
\hline Eczema & 1 \\
\hline Average Number Of CT Scan & 3.78 \\
\hline Interval Between The Adjacent Scans & 4.4 \\
\hline The Mulbsta Score & $7 \pm 3.46$ \\
\hline
\end{tabular}

creatinine in stage 3 was higher than the other two stage. Mean value of the ratio between ALB and GLB were lower than 1.5 in stage 1, 2 and 3. No increasing or decreasing of the average value of ALT and AST but the ratio of them (ALT/AST) was high than 1 in stage 1 and 3. Mean number of lymphocyte is in a downtrend while the eosinophil in an uptrend from stage 1 to stage 3 (Table 2, Figure 2).

\section{CT Imaging Features}

We missed patient $7 \mathrm{CT}$ scan in stage 1 for the reason we mentioned above. There was no CT scan of patient 3 


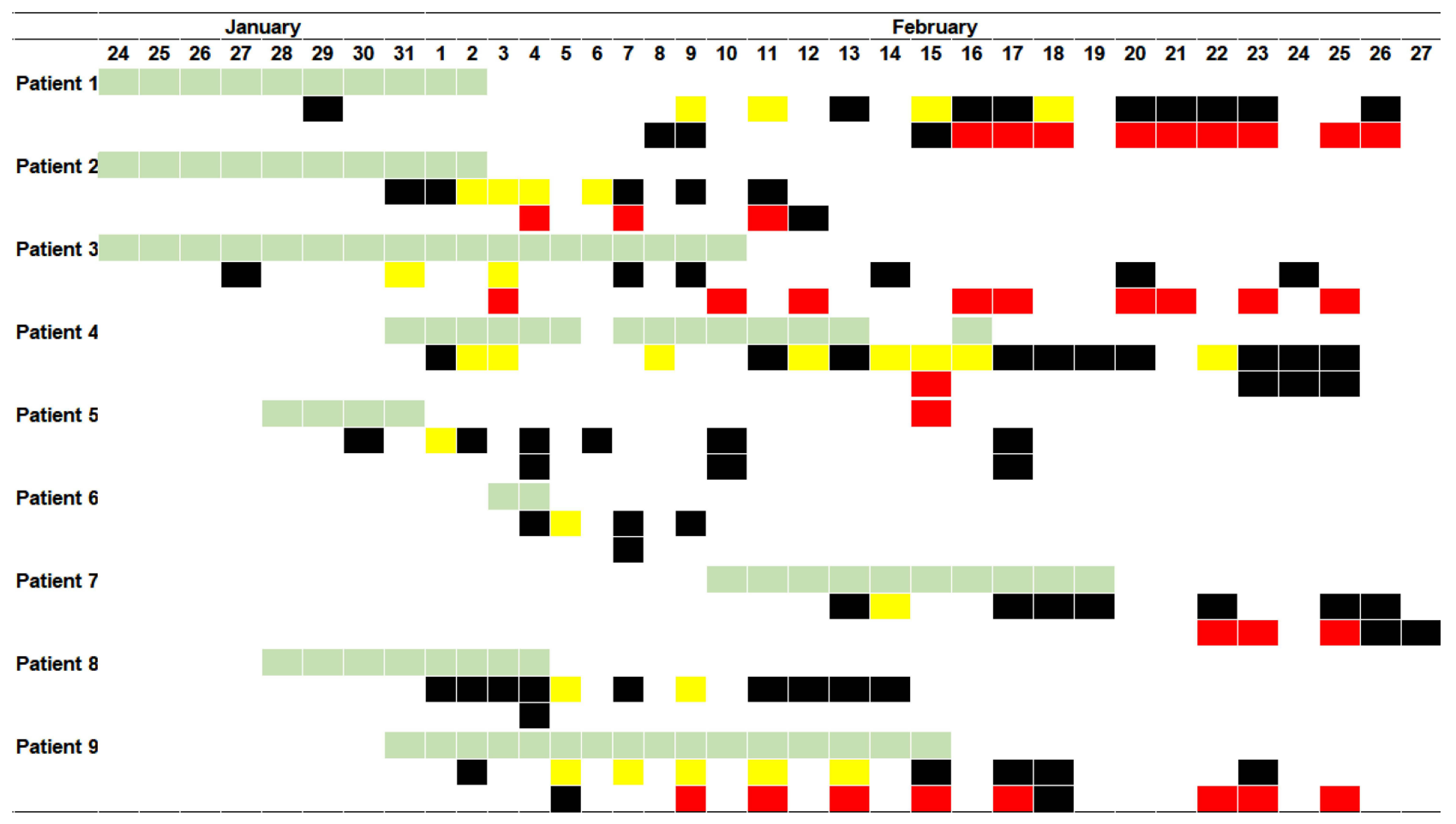

Figure I Timeline of symptoms onset and RT-PCR. Light green represents symptoms, yellow represents positive RT-PCR detection from respiratory samples, red represents positive RT-PCR detection from gastrointestinal samples, black represents negative RT-PCR detection.

and 8 during stage 2 , as well as patient 1 in stage 3 . The average interval of all $\mathrm{CT}$ scan was 4.4 days and mean number of CT scan was $1.22,1.67,1.11,3.78$ for stage $1,2,3$ and the whole, respectively. Two of the nine $(22.22 \%)$ had no lung lobe involved from stage 1 to stage 3 and three of the nine patients (33.33\%) had five lung lobes affected in all CT scan. Three of eight (37.5\%), 4/7 (57.14\%), 4/8 (50\%) of patients present bilateral multi-lobe involvement in stage 1, 2, 3 respectively. Lesions of two patients persisted in the left lower lobe. Six of the eight (75\%), 5/7 (71.43\%), 4/8 (50\%) of patients could found GGO in stage 1, 2, 3 respectively. Pleural effusion and absorbed were only seen in stage 3 $(2 / 8,25 \%)$. Only one of the patients showed central lesions and peripheral was the most common distribution (Table 3).

\section{Oxygen Support}

Two patients accepted oxygen support. Oxygen therapy by nasal tube were provided to patient 6 in stage 3 and patient 4 in stage 2. Patient 4 received a high-flow nasal cannula (HFNC) in stage 3 as the oxygenation index drop (Table 2).

\section{Discussion}

After Huang et al. reported COVID-19 in The Lancet, a number of studies has described the general epidemiology, clinical presentation, laboratory finding, radiological features of the confirmed cases including pregnant women and critically ill patients. ${ }^{5,8-10}$ Nowadays, RT-PCR provided by Chinese $\mathrm{CDC}$ is one of the major diagnosis standard. However, RT-PCR has limited sensitivity as well as depend much on the sample collecting, transporting, and kit performance. Anumber of COVID-19 cases were initially negative with RT-PCR detection. ${ }^{11}$ It provided the possibility of misdiagnosis or incorrect therapy and even made it a "super spreader". There is little research focusing on this part of people independently. Herein, our study presents the clinical characteristics of patients with firstly negative detection of COVID-19 nucleic acid.

Similar to the report in New England by Zhong, the age range of our patients is from 1 to 64 years, which indicates that all ages are susceptible to COVID-19. ${ }^{10}$

Consistent with other studies, fever and cough are predominant symptoms in stage 1 . However, no fever but cough persisted in stage 2 and only one case showed cough in stage 3. Screening for COVID-19 in the cough or other respiratory 
Table 2 Clinical Symptoms, Laboratory Findings and Oxygen Support

\begin{tabular}{|c|c|c|c|}
\hline & Stage I & Stage 2 & Stage 3 \\
\hline \multicolumn{4}{|l|}{ Symptoms } \\
\hline Fever & 6 & 0 & 0 \\
\hline Fatigue & 0 & 0 & 0 \\
\hline Cough & 5 & 3 & 1 \\
\hline Expectoration & 0 & 0 & 0 \\
\hline Chest Pain & 0 & 0 & 0 \\
\hline Chest Tightness & 2 & 2 & 0 \\
\hline Dyspnea & 0 & I & 0 \\
\hline Vomiting & 0 & 0 & 0 \\
\hline Poor Appetite & 0 & 0 & 0 \\
\hline Diarrhea & 1 & 0 & 0 \\
\hline Nausea & I & 0 & 0 \\
\hline \multicolumn{4}{|l|}{ Laboratory-Investigation } \\
\hline White Blood Cell Count & $5.46 \pm 1.99$ & $5.45 \pm 1.55$ & $6.15 \pm 2.53$ \\
\hline Neutrophil Count & $3.11 \pm 1.48$ & $2.94 \pm 1.27$ & $3.57 \pm 2.08$ \\
\hline Lymphocyte Count & $2.01 \pm 1.38$ & $1.94 \pm 0.9$ & $1.84 \pm 0.58$ \\
\hline Neutrophil/Lymphocyte & 1.55 & 1.52 & 1.94 \\
\hline Eosinophil Count & $0.08 \pm 0.1$ & $0.1 I \pm 0.11$ & $0.4 \pm 0.63$ \\
\hline C-Reactive Protein & $7.86 \pm 13.29$ & $11.88 \pm 24.47$ & $1.1 \pm 0.49$ \\
\hline Alanine Aminotransferase & $22.19 \pm|4.2|$ & $19.12 \pm 11.49$ & $34.6 I \pm 44.32$ \\
\hline Aspartate Aminotransferase & $19.31 \pm 7.59$ & $19.57 \pm 9.59$ & $26.14 \pm 23.65$ \\
\hline Alt/Ast & 1.15 & 0.98 & 1.32 \\
\hline Lactate & $1.55 \pm 0.49$ & $1.31 \pm 0.24$ & $1.71 \pm 0.52$ \\
\hline Serum Albumin & $38.93 \pm 2.47$ & $40.49 \pm 2.76$ & $39.66 \pm 2.82$ \\
\hline Seroglobulin & $28.94 \pm 3.33$ & $29.39 \pm 3.52$ & $30.39 \pm 4.95$ \\
\hline Alb/Glb & 1.35 & 1.38 & 1.31 \\
\hline Serum Creatinine & $52.54 \pm 17.27$ & $59.46 \pm 17.46$ & $64.66 \pm 9.21$ \\
\hline Creatine Kinase & $64.57 \pm 18.3$ & $58.11 \pm 14.79$ & $56.43 \pm 22.11$ \\
\hline Partial Arterial Oxygen Pressure & $105.7 \pm 8.49$ & $108.89 \pm 20.84$ & $|20.27 \pm 29.4|$ \\
\hline \multicolumn{4}{|l|}{ Oxygen Support } \\
\hline Noninvasive Ventilation & 0 & 0 & 0 \\
\hline High-Flow Nasal Cannula (HFNC) & 0 & 0 & I \\
\hline Nasal Tube & 0 & I & I \\
\hline Room Air & 9 & 8 & 7 \\
\hline
\end{tabular}

may be as important as in the fever. Noteworthy, patient 6 had no fever or symptoms associated to respiratory and his initial symptoms were nausea as well as diarrhea. Gastrointestinal symptoms were not common in COVID19 , affecting about $5 \%$ of people according to the data of 1099 patients. ${ }^{10}$ It indicates that COVID-19 symptoms are not limited to fever or respiratory issues and this should be taken into consideration with patients who have digestive ailments or other atypical symptoms.

The average number of the RT-PCR test is 1.44 days, indicating that a physician should repeat the RT-PCR test ton the suspected COVID-19 patients. Mean days of RTPCR from throat or nasal swab was positive after symptoms onset are 6.22 , longer the previous report and means days from fecal swab or stool cannot be calculated because this testing was only performed after COVID-19 confirmed. ${ }^{5}$ We noticed that average positive duration of feces is longer than upper respiratory (5.56 days vs. 6.56 days) and three patients' interval of stool even prolong half a month (until 28th February). There is little about the reason on the longtime of stool kept positive and it can even persist after throat or nasal swab converted negative. As reported, the gastrointestinal tract showed an underlying infection route of COVID-19 in pre-print work, thus, we suggest a gastrointestinal sample should be tested routinely. $^{2}$ 


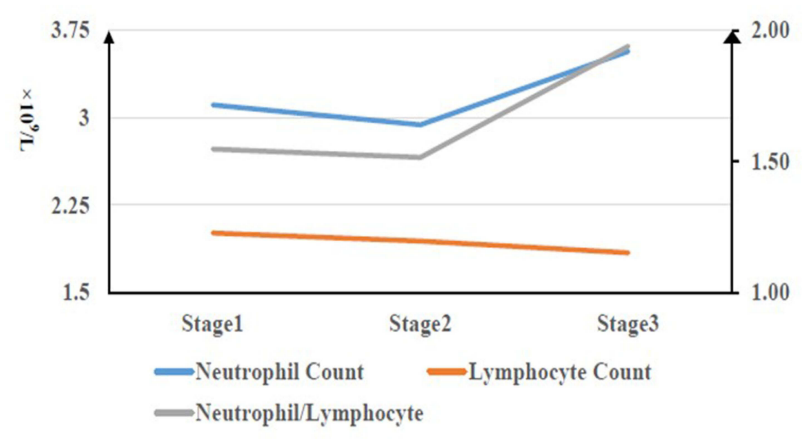

A

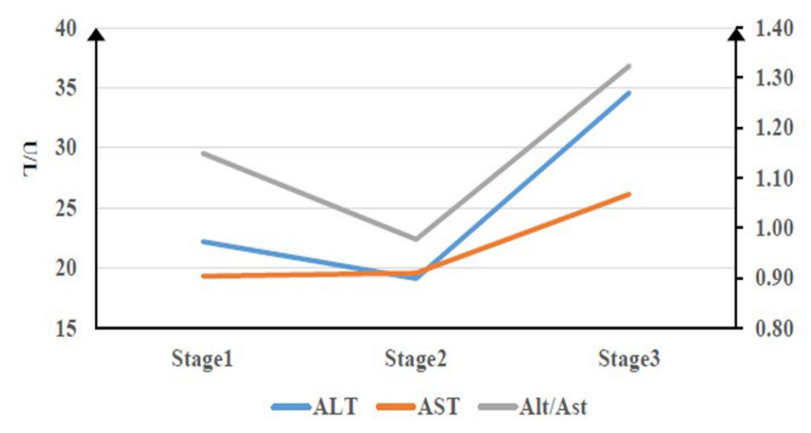

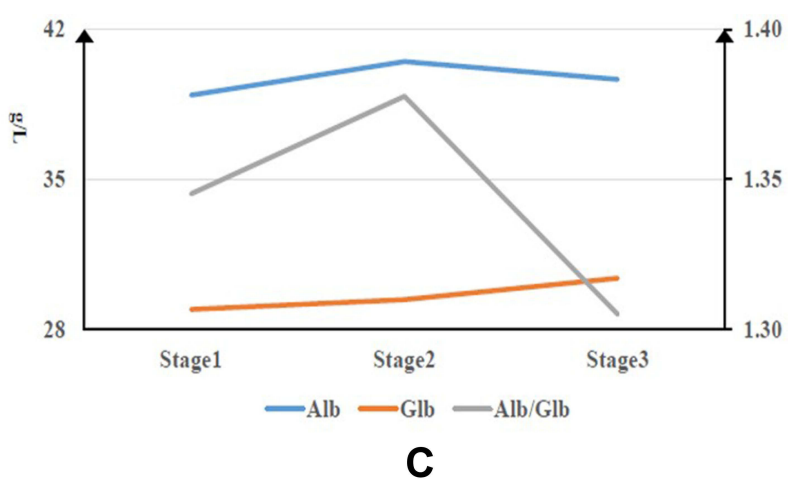

B

Figure 2 Timeline charts illustrate the mean laboratory parameters in various stages. On the left primary vertical axis is the quantitative variable and on the right secondary axis is the ratio. (A) Neutrophil Count, Lymphocyte Count and ratio of them among each stage. (B) ALT, AST and ratio of them among each stage. (C) Alb, Glb and ratio of them among each stage.

Regarding laboratory tests, the pro-inflammatory effect probably contribute to the reasons why the lymphocyte count dropped from stage 1 to $3 .^{12}$ A pathological reports exhibited that moderate micro vesicular steatosis and mild lobular and portal activity in the liver with COVID-19, which indicated that SARS-CoV probably damaged the liver. There is researchabout liver dysfunction in COVID-19. One patient presented with high ALT and nearly half of the patients presented with low ALB from stage 1 to 3 . Additionally, although the ALT as well as AST were at normal levesl, the ratios between ALT and AST were high than 1 in stage 1 and stage 3. These laboratory findings may indicate SARS-CoV was able to cause imperceptible liver damage and mechanism needs to be explored in the further.

Regarding the CT scan, similar to other researches, we found that multiple-lobes involvement and peripheral distribution were common. However, in the present study, instead of right low lobe, left low lobe is affected mostly., Consistent with a recent report which suggested that more than $70 \%$ COVID-19 patients with negative RT-PCR results had typical CT manifestations, 6/8 (75\%) of our patients' CT scans had GGO, a typical symbol of viral pneumonia. Two patients were negative in CT scans during the whole hospitalization, which reveals that repeated RT-PCR test is irreplaceable. A CT scan was usually performed according to the symptoms, as well laboratory findings of patients and physician and we recommend that chest CT should be performed as a supplement to RT-PCR during the COVID-19 outbreak.

Mulbsta score is easy-to-use clinically predictive tool for assessing 90-day mortality risk. 0-11 means "low-risk" (mortality $=5.07 \%$ ) and 12-22 means "high-risk" (mortality $=33.92 \%)^{7}$ Oxygen index of patient 4 with a high risk score (score 13) deteriorated during the treatment. Thanks to this scoring system, he received oxygen support including by nasal tube and HFNC quickly. The scoring system enhances the confidence of severity evaluation in physician. We suggest that it should performed among all the COVID-19 patients if possible.

Our study is limited by the small sample and some of the patients are still hospitalized or persist with positive stool detection at the time of writing, which means we cannot evaluate the factors related to outcome, such as the duration of positive detection, oxygen support lasting time or Mulbsta score. Moreover, there is increasing SARSCoV-2 variants but our study only focus on the early strain 
Table 3 CT Scan Features

\begin{tabular}{|c|c|c|c|}
\hline & Stage I & Stage 2 & Stage 3 \\
\hline \multicolumn{4}{|l|}{ Number of Involved Lobes } \\
\hline 0 & 2 & 2 & 2 \\
\hline I & 2 & 2 & 2 \\
\hline 2 & I & I & I \\
\hline 3 & 0 & I & 0 \\
\hline 4 & 0 & 0 & I \\
\hline 5 & 3 & 2 & 2 \\
\hline \multicolumn{4}{|l|}{ Frequency of Lobes } \\
\hline \multicolumn{4}{|l|}{ Abnormalities } \\
\hline Left Upper & 4 & 3 & 3 \\
\hline Left Lower & 5 & 6 & 6 \\
\hline Right Upper & 3 & 2 & 3 \\
\hline Right Middle & 3 & 2 & 2 \\
\hline Right Lower & 4 & 4 & 4 \\
\hline Only Left & 2 & 2 & 2 \\
\hline Only Right & 0 & 0 & 0 \\
\hline Bilateral Multilobe & 3 & 4 & 4 \\
\hline \multirow{2}{*}{\multicolumn{4}{|c|}{$\begin{array}{l}\text { Distribution of Involved } \\
\text { Lobes }\end{array}$}} \\
\hline & & & \\
\hline Central & 0 & 1 & I \\
\hline Peripheral & 5 & 5 & 5 \\
\hline Random & I & 0 & 0 \\
\hline Diffuse & 0 & 0 & 0 \\
\hline GGO & 5 & 5 & 4 \\
\hline Crazy-Paving Pattern & 0 & I & 2 \\
\hline Consolidation & 0 & 1 & I \\
\hline Effusion & 0 & 0 & 2 \\
\hline Absorbed & 0 & 0 & 3 \\
\hline
\end{tabular}

as the data was collected at the beginning of COVID-19 outbreak (18th January to 26th February, 2020). Thus, maybe there are different features among the variants.

\section{Limitation}

Our study was performed in the early stage of COVID-19 outbreak and thus the sample size may be unsatisfactory.

\section{Conclusion}

In conclusion, our study elucidated and gave insight on the clinical features of hospitalized patients with initially negative detection of COVID-19 nucleic acid. Notably, we suggest that patient with only symptoms of digestive system issues should screen for SARS-CoV-2, like the one with fever or respiratory symptoms. CT scan and repeated
RT-PCR for COVID-19 are extremely necessary. Mulbsta score should performed as routine work to the COVID-19.

\section{Data Sharing Statement}

The data sets generated and analyzed during the present study are available from the corresponding author (Jing Liu) on reasonable request.

\section{Ethical Approval and Consent to Participate}

Our study was approved by the Research Ethics Committee of the Fifth Affiliated Hospital of Sun Yatsen University (Ethics approval number K30-1).

\section{Consent for Publication}

All authors have read and approved the manuscript.

\section{Funding}

This work was supported by Guangdong Basic and Applied Basic Research Foundation (2020A1515011147) and Zhuhai Science and Technology Project (ZH22036 302200021PWC).

\section{Disclosure}

The authors report no conflicts of interest in this work.

\section{References}

1. Zhu N, Zhang D, Wang W, et al. A Novel Coronavirus from Patients with Pneumonia in China, 2019. N Engl J Med. 2020;382(8)727-733.

2. Zhang H, Kang Z, Gong H, et al. The digestive system is a potential route of 2019-nCov infection: a bioinformatics analysis based on single-cell transcriptomes. bioRxiv. 2020;2020-2021. doi:10.1101/ 2020.01.30.927806

3. Zhou P, Yang XL, Wang XG, et al. A pneumonia outbreak associated with a new coronavirus of probable bat origin. Nature. 2020;588:E6E6. doi:10.1038/s41586-020-2951-z

4. Huang C, Wang Y, Li X, et al. Clinical features of patients infected with 2019 novel coronavirus in Wuhan, China. Lancet. 2020;395 (10223):497-506. [[Journal Article; Research Support, Non-U.S. Gov't]]. doi:10.1016/S0140-6736(20)30183-5

5. Bernheim A, Mei X, Huang M, et al. Chest CT findings in coronavirus disease-19 (COVID-19): relationship to duration of infection. Radiology. 2020;295(3):200463. doi:10.1148/radiol.2020200463

6. Pan F, Ye T, Sun P, et al. Time course of lung changes on chest CT during recovery from 2019 novel coronavirus (COVID-19) pneumonia. Radiology. 2020;200370. doi:10.1148/radiol.2020200370

7. Guo L, Wei D, Zhang X, et al. Clinical features predicting mortality risk in patients with viral pneumonia: the MuLBSTA score. Front Microbiol. 2019;10:2752. doi:10.3389/fmicb.2019.02752

8. Wang X, Zhou Z, Zhang J, Zhu F, Tang Y, Shen X. A case of 2019 Novel Coronavirus in a pregnant woman with preterm delivery. Clin Infect Dis. 2020;71(15):844-846. doi:10.1093/cid/ciaa200 
9. Wang D, Hu B, Hu C, et al. Clinical characteristics of 138 hospitalized patients with 2019 novel coronavirus-infected pneumonia in Wuhan, China. JAMA. 2020;323:1061.

10. Guan WJ, Ni ZY, Hu Y, et al. Clinical characteristics of coronavirus disease 2019 in China. $N$ Engl J Med. 2020;382:1708-1720. doi:10.1056/NEJMoa2002032

11. Ai T, Yang Z, Hou H, et al. Correlation of Chest CT and RT-PCR Testing in Coronavirus Disease 2019 (COVID-19) in China: a Report of 1014 Cases. Radiology. 2020;296:E32.
12. Peiris JS, Chu CM, Cheng VC, et al. Clinical progression and viral load in a community outbreak of coronavirus-associated SARS pneumonia: a prospective study. Lancet. 2003;361 (9371):1767-1772. [[Journal Article; Research Support, NonU.S. Gov't; Research Support, U.S. Gov't, P.H.S.]]. doi:10.10 16/S0140-6736(03)13412-5

\section{Publish your work in this journal}

The International Journal of General Medicine is an international, peer-reviewed open-access journal that focuses on general and internal medicine, pathogenesis, epidemiology, diagnosis, monitoring and treatment protocols. The journal is characterized by the rapid reporting of reviews, original research and clinical studies across all disease areas. The manuscript management system is completely online and includes a very quick and fair peer-review system, which is all easy to use. Visit http://www.dovepress.com/ testimonials.php to read real quotes from published authors. 